\title{
Matrine inhibits the proliferation of pituitary tumor cells by decreasing Foxo3a phosphorylation and promoting Foxo3a nuclear localization
}

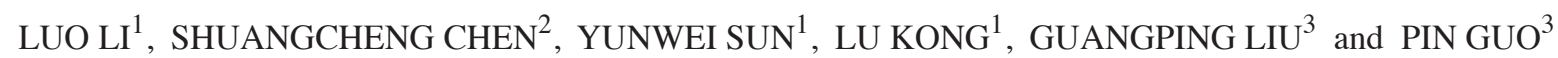 \\ ${ }^{1}$ Department of Neurosurgery, Qingdao Municipal Hospital, Qingdao University, Qingdao, Shandong 266000; \\ ${ }^{2}$ Department of Neurosurgery, RenMin Hospital of Jimo, Qingdao, Shandong 266200; ${ }^{3}$ Department of Neurosurgery, \\ The Affiliated Hospital of Qingdao University, Qingdao, Shandong 266000, P.R. China
}

Received November 13, 2017; Accepted September 28, 2019

DOI: $10.3892 /$ etm.2019.7365

\begin{abstract}
Pituitary tumors account for $10 \%$ of intracranial cancer, and are difficult to treat with chemotherapy. The aim of the present study was to explore the role of matrine on the proliferation of pituitary cancer cells, as well as the molecular mechanism of matrine in progression and development of pituitary tumors. Matrine significantly suppressed the proliferation of pituitary cancer cells in a dose- and time-dependent manner. Western blotting results showed that the phosphorylation levels of AKT serine/threonine kinase (Akt) and forkhead box O3A (Foxo3a) decreased as the concentration of matrine increased. Matrine increased the nuclear localization of Foxo3a and the expression of proapoptotic genes, such as BCL2 like 11 and BCL2 associated X apoptosis regulator, and inhibited the levels of cytoplasmic Foxo3a. In conclusion, matrine promoted cell death of pituitary cancer cells and was involved in Akt/Foxo3a signaling pathway.
\end{abstract}

\section{Introduction}

Pituitary tumors account for $10 \%$ of intracranial cancer, which is derived from the remnant cells in anterior pituitary, posterior pituitary and craniopharyngeal epithelium, and represents $10-15 \%$ of all intracranial tumors. Each patient with pituitary cancers should be given individually selected treatment approach (1). Normally, pituitary surgery is one of the biggest series including

Correspondence to: Professor Pin Guo, Department of Neurosurgery, The Affiliated Hospital of Qingdao University, 16 Jiangsu Road, Shinan, Qingdao, Shandong 266000, P.R. China E-mail: guopinqingdao@hotmail.com

Dr Luo Li, Department of Neurosurgery, Qingdao Municipal Hospital, Qingdao University, 5 Donghaizhong Road, Shinan, Qingdao, Shandong 266000, P.R. China

E-mail: liluoqd@163.com

Key words: pituitary cancer, matrine, Akt, Foxo3a, nuclear localization transcranial, transsphenoidal, and endoscopic techniques. Transsphenoidal surgery is usually considered as the first-choice therapy for most patients with pituitary tumors, which is an effective and safe treatment (2). Comprehensive therapy is gradually important among endocrinologists, ophthalmologists, and radiologists (3). Thus, it is helpful to clarify the pathogenesis and progression of pituitary tumors. The newly discovered reports are important to give us a clue to clarify the molecular mechanism of pituitary tumors. For example, histone deacetylases (HDAC) 11 had a key function in multiple cellular activities and HDAC11 suppressed P53 expression in pituitary tumor cells. Inhibition of HDAC11 had a therapeutic potential in the treatment of pituitary tumors (4). MiRNAs were involved in regulating the progression of pituitary tumors. MiRNA153 inhibited MMQ cell growth and promoted cell apoptosis by down-regulating Skp protein (5). Enhancer of zeste homolog $2(\mathrm{EZH} 2)$ levels were associated with the invasiveness, proliferation, angiogenesis, hormone function in pituitary adenomas, which could be a useful diagnostic marker and pharmacotherapeutic target for malignant pituitary tumors (6). Thus, it is important to demonstrate the pathogenesis and progression of pituitary tumors and find an effective antitumor drug candidate for pituitary tumors.

To date, Chinese herb are widely used for thousands of human cancers in clinical therapy. Matrine is a major active compound and extracted from the dry roots of Sophora flavescens, which has been reported to possess potent antitumor activity in various human cancer cells. Matrine inhibited cell proliferation and induced apoptosis of human colon cancer cells in vitro by upregulating Bax, and downregulating $\mathrm{Bcl}-2$ and releasing $\mathrm{Cyto} \mathrm{C}$ from the mitochondria to the cytosol and activating caspase- 3 and caspase- 9 (7). This was consistent with the founding in breast cancers, which was found that matrine inhibited the growth and induced apoptosis of breast carcinoma MCF-7 cells (8). Moreover, matrine showed the suppression activity in the proliferation and metastasis of highly-metastatic breast cancer MDA-MB-231 cells via EGF/VEGF-VEGFR1-Akt-NF-kappaB signaling pathway (9). Matrine suppressed gastric cancer cell line MNK45 in a dose-dependent manner and the anti-tumor activity was associated with the modulation of the NF-kappaB, XIAP, CIAP, and p-ERK proteins expression in MNK45 
cells $(10,11)$. Autophagy, a new identified programmed cell death, played an important role in tumor progression. Zhang, J. found that matrine suppressed the proliferation of SGC-7901 gastric cancer cells and induced G1-phase cell cycle arrest by activating both autophagy and apoptosis in the therapy of gastric cancers (12). The microRNAs, a class of small, non-coding, regulatory RNAs were reported to be involved in the tumorigenesis of human cancers. In gastric cancer cell line SGC7901, matrine treatment induced considerable changes in the miRNA expression profiles of SGC7901 cells, which was involved in 57 identified enrichment pathways in tumorigenesis (13). In non-small lung cancer cell lines, matrine induced the cell arrest at G1/G0 phase and cell apoptosis by uperegulating the expression of miR126 (14).

Matrine, an alkaloid extracted from the Chinese herb Sophora flavescens Ait, may be a promising candidate drug in the therapy of human various cancers. However, the molecular mechanisms of matrine in pituitary cancer cells was not clearly clarified and the involved anti-tumor mechanism need to be further explored.

\section{Materials and methods}

Cell lines and agents. The mouse pituitary tumor cell line AtT-20 (CRL1795, ATCC) and rat pituitary GH3 tumor cell line RC-4B/C (cat. no: JN-C0859; Rongbai biological corporation, Shanghai, China) were cultured in Ham's F-10 nutrient mixture containing with $15 \%$ horse serum (cat. no: 26050070 ) and $2.5 \%$ fetal bovine serum (cat. no: 10099141), which were purchased from Thermo Fisher Scientific, Inc., Waltham, MA, USA. Rat pituitary tumor cells (cat. no: GH3 FS-C-0014) was purchased from Fengshou biological corporation (Shanghai, China). The cells were cultured in DMEM medium (Invitrogen; Thermo Fisher Scientific, Inc.) with $10 \%$ fetal bovine serum (Trace Scientific Ltd., Melbourne, Australia) at $37^{\circ} \mathrm{C}$ in an atmosphere of $95 \%$ air and $5 \% \mathrm{CO}_{2}$. Matrine (cat. no: HY-N0164) was obtained from MedChemExpress (Shanghai, China) with purity of more than $99.80 \%$. MTT agent was obtained from Sigma-Aldrich; Merck KGaA (Darmstadt, Germany).

MTT assay. Cell viability was determined by MTT assay. Briefly, the pituitary tumor cells pituitary $\mathrm{RC}-4 \mathrm{~B} / \mathrm{C}$ and rat pituitary tumor cells GH3 were plated into 96-well plate and cultured for $6 \mathrm{~h}$. The cells were treated with different dose of matrine for $24 \mathrm{~h}$. The concentrations of matrine were 0.1 , 0.5 and $2.5 \mathrm{mg} / \mathrm{ml}$, respectively. Matrine was dissolved with DMSO and the final concentration of DMSO in medium was $0.1 \%$. The cells treated with $0.1 \%$ DMSO were used as negative controls. In the other experiment, RC-4B/C cells and $\mathrm{GH} 3$ cells were treated with $1.0 \mathrm{mg} / \mathrm{ml}$ of matrine for 24,48 and $72 \mathrm{~h}$, respectively. The cell viability was determined MTT assay and the survival rate of matrine-treated pituitary cancer cells was calculated by graphpad 5.0.

Nuclear and cytoplasmic protein extraction. The RC-4B/C cells were treated with different concentrations of matrine for $24 \mathrm{~h}$. The total cells in each group were collected. The nuclear and cytoplasmic proteins were extracted by nuclear and cytoplasmic protein extraction kit (cat. no: P0027), which was purchased from Beyotime Institute of Biotechnology,
(Haimen, China). The levels of cytoplasmic and nuclear of Foxo3a was detected by western blotting. Here, $\beta$-tublin was used as the marker in cytoplasm and lamin B1 was used as the internal reference in nucleus.

Western blot analysis. The western blotting was performed as described $(15,16)$. The levels of p-Akt, total Akt, p-FoxO3a, total Foxo3a, Bim, Bax and Bcl-2 were detected by western blotting analysis. The antibodies used here were shown as follows: FoxO3a (75D8; cat. no: \#2497) was a rabbit monoclonal antibody for FoxO3a protein and purchased from Cell Signaling Technology, Inc., (Danvers, MA, USA). The p-Akt antibody (cat. no: sc-7985-R, a rabbit polyclonal $\mathrm{IgG}$ ) and Akt antibody (BDI111, cat. no: sc-56878, a mouse monoclonal IgG1) was purchased from Santa Cruz Biotechnology, Inc., (Dallas, TX, USA). Anti-Bim antibody [Y36] (ab32158) was a rabbit monoclonal IgG and purchased from Abcam Corporation. Bax (D2E11) rabbit mAb no: 5023 was a rabbit IgG and detected endogenous levels of total Bax protein, which was obtained from Cell Signaling Technology, Inc. Anti-Bcl-2 antibody [E17] (ab32124) was a rabbit monoclonal antibody and purchased from Abcam corporation. $\beta$-actin Antibody (C4) (cat. no: sc-47778) was a mouse monoclonal IgG1 and purchased from Santa Cruz Biotechnology, Inc. The 'grey values' was determined from scans of western blots according to software protocols. The software used for densitometry is Image J (v1.48; National Institutes of Health, Bethesda, MD, USA).

Statistical analysis. The data was analyzed with SPSS software v13 (SPSS Inc., Chicago, IL, USA). In the present study, two sets of independent samples were analyzed with the student's t-test. The multiple comparisons were analyzed by using ANOVA and Tukey test. The data was analyzed with t-test. The results were shown as mean \pm standard deviations. $\mathrm{P}<0.05$ was considered to indicate a statistically significant difference.

\section{Results}

Matrine inhibits cell proliferation of pituitary cancer cells. Firstly, we wanted to test whether matrine had inhibitory effects on the proliferation of pituitary cancer cells. The pituitary cancer cells $\mathrm{RC}-4 \mathrm{~B} / \mathrm{C}$ and $\mathrm{GH} 3$ were treated with increasing concentrations of matrine for $24 \mathrm{~h}$ and MTT assay was performed to detect cell viability of matrine-treated pituitary cancer cells. As shown in Fig. 1A, the survival rates of RC-4B/C cells and $\mathrm{GH} 3$ cells were significantly decreased in matrine-treated cells than that of $0.1 \%$ DMSO-treated cells $\left({ }^{*} \mathrm{P}<0.05,{ }^{* *} \mathrm{P}<0.01\right.$, compared with $0.1 \%$ DMSO treated pituitary $\mathrm{RC}-4 \mathrm{~B} / \mathrm{C}$ cells and $\mathrm{GH} 3$ cells). There was no statistical difference between the cells treated with $0.1 \mathrm{mg} / \mathrm{ml}$ of matrine and $0.1 \%$ DMSO. Next, $1.0 \mathrm{mg} / \mathrm{ml}$ of matrine were used to treat pituitary cancer cells for 24,48 and $72 \mathrm{~h}$, respectively. As shown in Fig. 1B, the survival rates of $\mathrm{RC}-4 \mathrm{~B} / \mathrm{C}$ cells and $\mathrm{GH} 3$ cells significantly decreased in a time-dependent manner $\left({ }^{*} \mathrm{P}<0.05,{ }^{* *} \mathrm{P}<0.01\right.$, compared with the survival rate in $24 \mathrm{~h}$ ). All the data revealed that matrine inhibited cell proliferation of pituitary cancer cells in a dose- and time-dependent manner.

Matrine inhibits the phosphorylation levels of Akt and Foxo3a in $R C-4 B / C$ cells. Next, we further investigated the molecular 
A

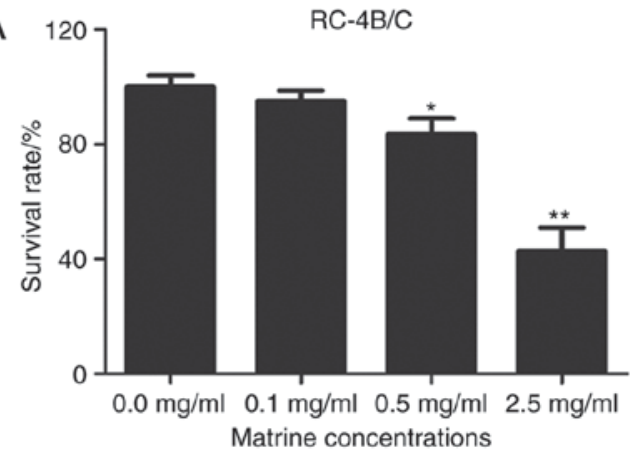

B

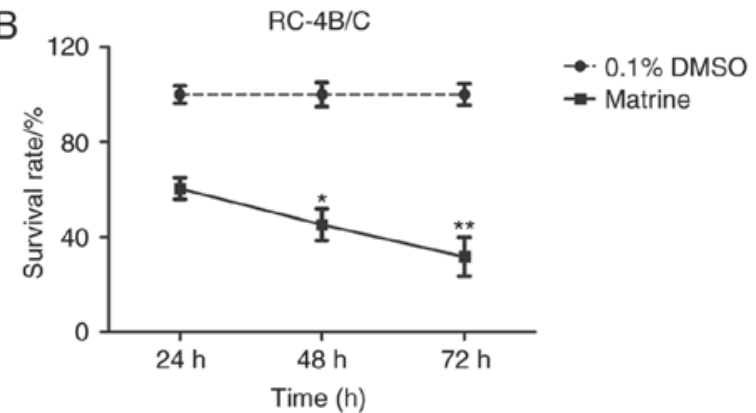

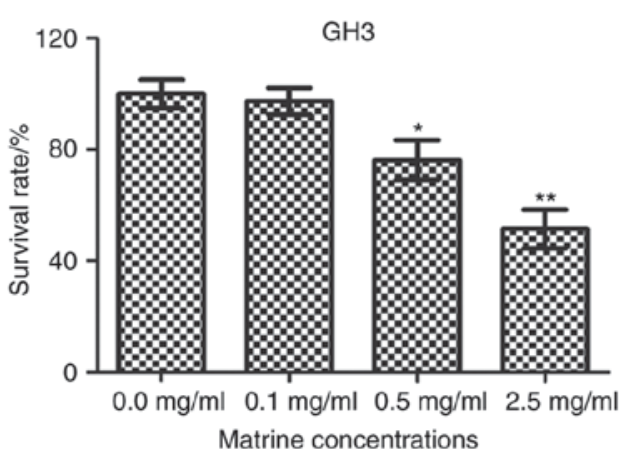

$\mathrm{GH} 3$

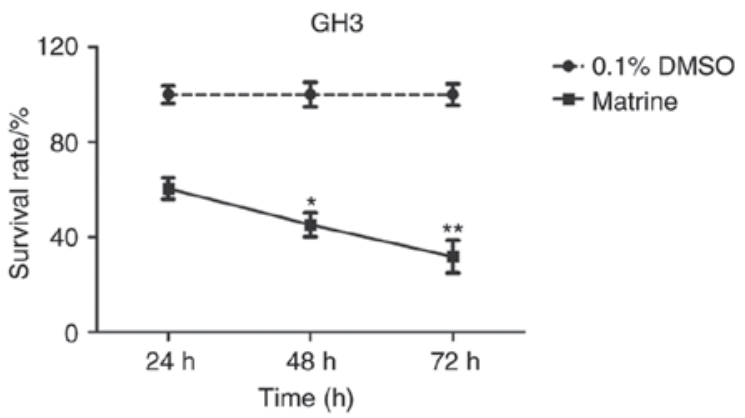

Figure 1. Matrine inhibits cell proliferation of pituitary cancer cells. (A) The pituitary cancer cells RC-4B/C and GH3 were treated with increasing concentrations of matrine for $24 \mathrm{~h}$. The concentration of matrine was $0.1,0.5$ and $2.5 \mathrm{mg} / \mathrm{ml}$. The cells treated with $0.1 \%$ DMSO was used as negative controls. MTT assay was performed to detect cell viability of matrine-treated pituitary cancer cells. ${ }^{*} \mathrm{P}<0.05,{ }^{* *} \mathrm{P}<0.01$, compared with $0.1 \% \mathrm{DMSO}$ treated cells. (B) RC-4B/C cells were treated with $1 \mathrm{mg} / \mathrm{ml}$ of matrine for 24,48 and $72 \mathrm{~h}$, respectively. The cell viability was determined by MTT assay. The survival rate in each group was shown in line chart. ${ }^{*} \mathrm{P}<0.05,{ }^{* *} \mathrm{P}<0.01$, compared with matrine treated group in $24 \mathrm{~h}$.

mechanism that how matrine possessed the anti-tumor effects in $\mathrm{RC}-4 \mathrm{~B} / \mathrm{C}$ cells. Western blotting analysis was used to test whether matrine affected Akt signaling pathway. Briefly, $\mathrm{RC}-4 \mathrm{~B} / \mathrm{C}$ cells were treated with $0.1,0.5$ and $2.5 \mathrm{mg} / \mathrm{ml}$ of matrine for $24 \mathrm{~h}$ and the cells treated with $0.1 \%$ DMSO were used as negative controls. The levels of phosphorylated Akt, total Akt, phosphorylated Foxo3a and total Foxo3a were tested by western blotting analysis. As shown in Fig. 2, as the concentration of matrine increased, the expression of $\mathrm{p}-\mathrm{Akt}$ and p-Foxo3a significantly decreased, while the levels of total Akt and total Foxo3a were not obviously changed. All the data showed that matrine regulated the proliferation of pituitary cancer cells probably by Akt/Foxo3a signaling pathway.

Matrine promotes the nuclear Foxo3a localization and inhibits the levels of cytoplasmic Foxo3a. FOXO3A, a transcription factors of the forkhead family, was phosphorylated and inactivated via PI3K/Akt signaling pathway. Next, we detected whether matrine induced the re-localization of Foxo3a in $\mathrm{RC}-4 \mathrm{~B} / \mathrm{C}$ cells. The cytoplasmic and nuclear proteins were extracted in matrine-treated RC-4B/C cells, as shown in Fig. 3, the levels of Foxo3a in nucleus gradually increased as the concentration of matrine increased in $\mathrm{RC}-4 \mathrm{~B} / \mathrm{C}$ cells, while the cytoplasmic Foxo3a level significantly decreased, suggesting that matrine treatment promoted the nuclear localization of Foxo3a by inhibiting the phosphorylation of Foxo3a.

Matrine increases the expression of pro-apoptotic proteins in $R C-4 B / C$ cells. It has reported that activated Foxo3a promoted cell apoptosis through expression of genes for cell death, such as Fas ligand (FasL), Bim and tumor necrosis factor related apoptosis-inducing ligand (TRAIL) etc. Then, we tested the expression of apoptosis related proteins in matrine-treated pituitary cancer cells. As shown in Fig. 4, RC-4B/C cells were treated with $0.1,0.5$ and $2.5 \mathrm{mg} / \mathrm{ml}$ of matrine for $24 \mathrm{~h}$, and the levels of Bim, Bax and Bcl-2 were determined by western blotting analysis. The results demonstrated that matrine promoted the expression of Bim and increased the ratio of $\mathrm{Bax} / \mathrm{Bcl}-2$.

\section{Discussion}

Pituitary cancer is one of the genitourinary malignancies with high mortality that seriously threatens human health. The new approaches and drugs are being developed to improve quality of pituitary cancer patients. One of the important way to concur pituitary cancers is to clarify the mechanism of tumorigenesis and proliferation of pituitary cancer cells. In the present study, we explored the molecular mechanism of matrine in pituitary cancer cells, which could be used as an effective drug candidate in the clinical therapy of pituitary cancers.

Firstly, the anticancer activity of matrine in pituitary cancer cells were tested by MTT assay. We found that matrine inhibited cell viability of $\mathrm{RC}-4 \mathrm{~B} / \mathrm{C}$ cells and $\mathrm{GH} 3$ cells in a time-dependent and dose-dependent manner. The results demonstrated that matrine had potent antitumor activity in pituitary cancer cells. This was consistent with the results that matrine suppressed bladder tumor invasion in a rat model, which could be primarily mediated by regulating the expression of COX-2 and cPLA2 in the bladder (17). Furthermore, we explored the molecular mechanism of matrine in pituitary cancer cells by western blotting analysis. It has been reported that matrine regulated the cell proliferation of cancer cells by 


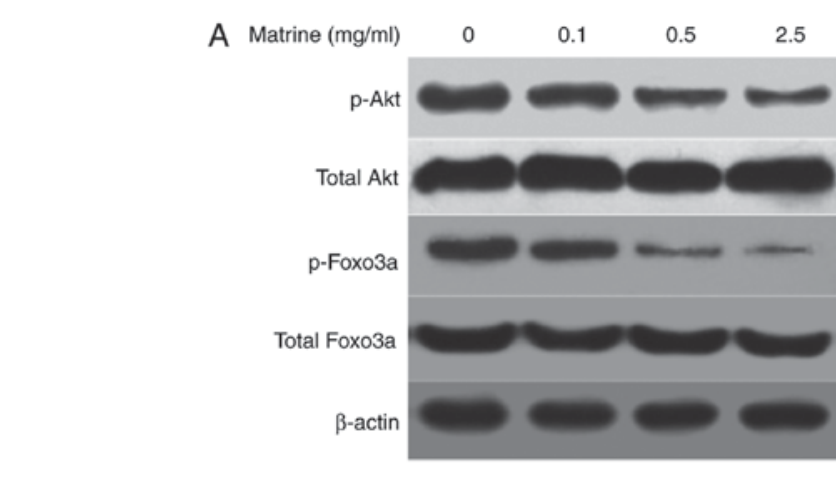

B
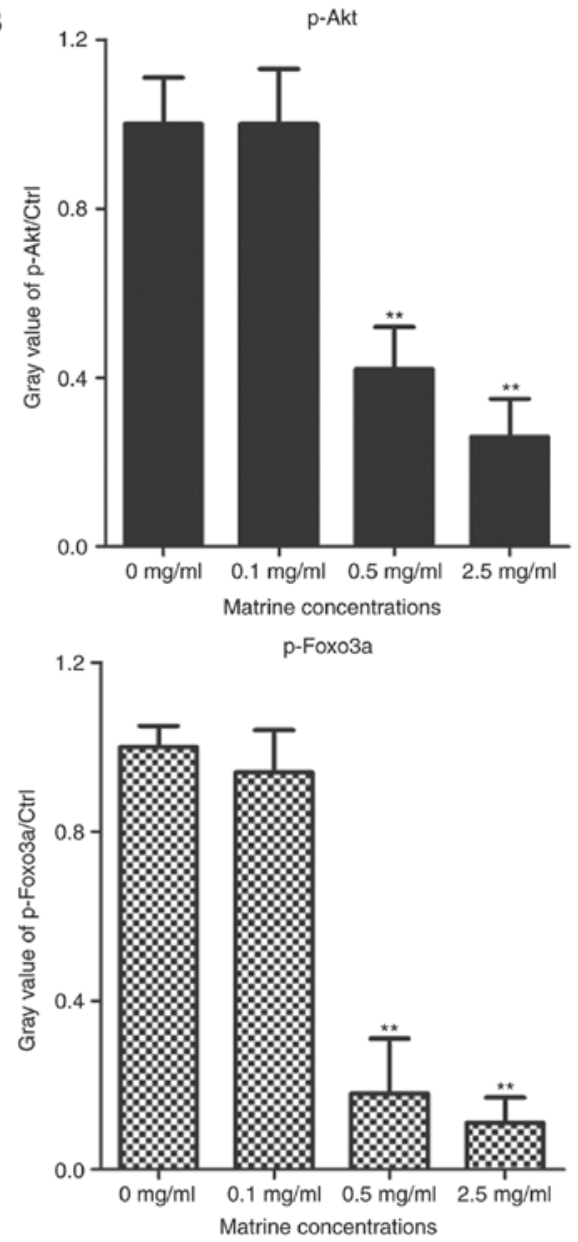

Total Akt

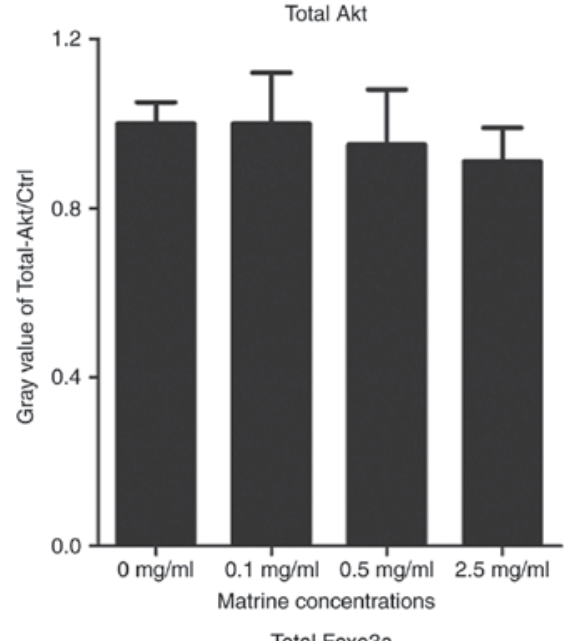

Total Foxo3a

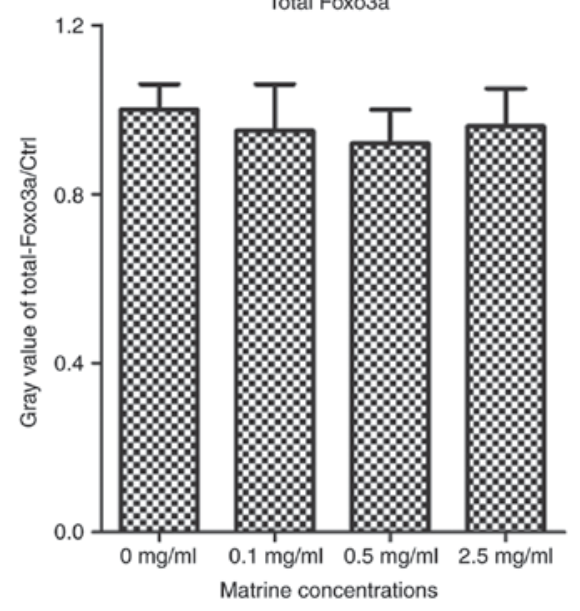

Figure 2. Matrine inhibits the phosphorylation levels of Akt and Foxo3a in RC-4B/C cells. (A) RC-4B/C cells were treated with matrine at the concentration of $0,0.1,0.5$ and $2.5 \mathrm{mg} / \mathrm{ml}$ for $24 \mathrm{~h}$. The levels of p-Akt, total Akt, p-Foxo3a and total Foxo3a were determined by western blotting analysis. $\beta$-actin was used as internal reference gene in pituitary cancer cells. (B) The gray values of p-Akt, total Akt, p-Foxo3a and total Foxo3a were shown in histogram in matrine-treated RC-4B/C cells. "* $\mathrm{P}<0.01$, compared with the pituitary cancer cells without matrine. p-Akt, phosphorylated Akt; p-Foxo3a, phosphorylated Foxo3a.

suppressing PI3K/Akt signaling pathways (18,19), Akt/Foxo pathway (20), Akt/m TOR/S6K and ERK1/2 signaling (21), which was detected in pituitary cancer cells. Then, we tested whether matrine was involved in regulating Akt/Foxo3a signaling pathway in the cell proliferation of pituitary cancer cells. The phosphorylated Foxo3a was translocated into cytoplasm and inhibited the down-stream gene expression, which inhibited cell apoptosis in various cancer cells. Luckily, the results demonstrated that as the concentration of matrine increased, the levels of p-Akt significantly decreased, as well as the levels of pFoxo3a in matrine-treated RC-4B/C cells. Moreover, we extracted the total nuclear and cytoplasmic proteins of matrine-treated RC-4B/C cells and tested the levels of Foxo3a by western blotting analysis. This result revealed that as the concentration of matrine increased, the level of Foxo3a in cytoplasm significantly decreased and the level of foxo3a in nucleus obviously increased. This was consistent with the expression of the downstream gene, bim, which obviously increased as the concentration of matrine increased in RC-4B/C cells.

However, there was a limitation in our study that we did not successfully identify and isolate the normal counterpart of pituitary tumor cells. Thus, we does not evaluate the matrine on normal pituitary tissue, and it remains unclear whether 
A

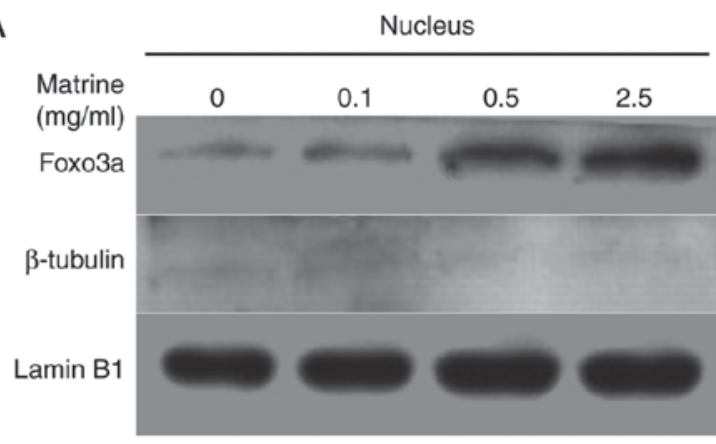

Nuclear Foxo3a

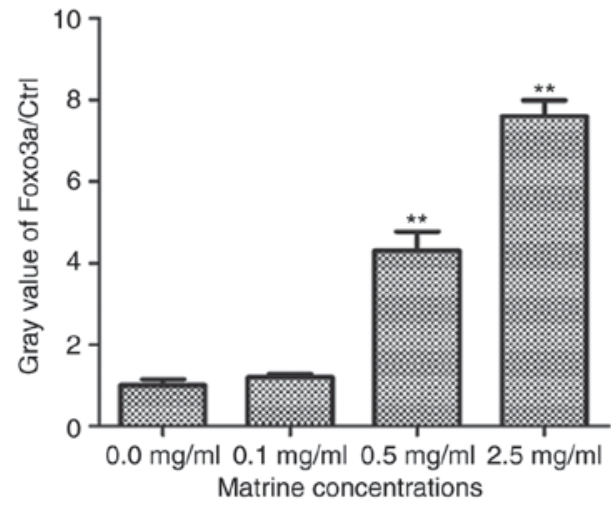

B

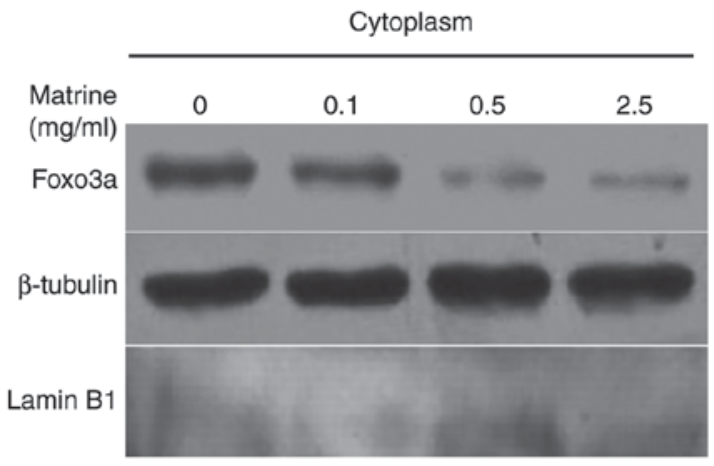

Cytoplasmic Foxo3a

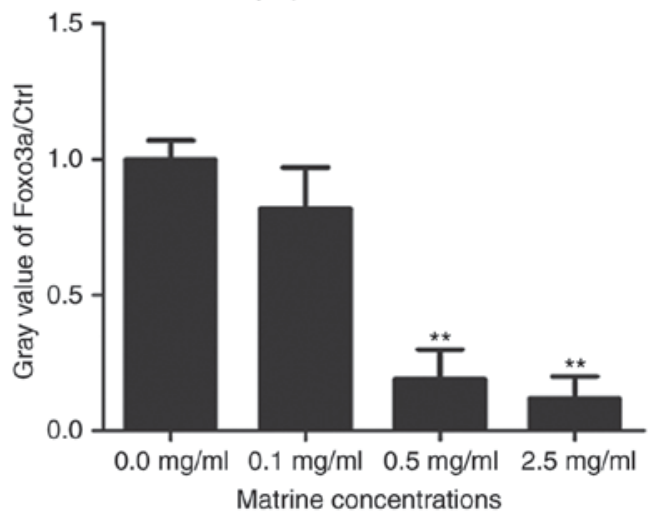

Figure 3. Matrine promotes the nuclear Foxo3a localization and inhibits the levels of cytoplasmic Foxo3a. RC-4B/C cells were treated with increasing concentrations of matrine for $24 \mathrm{~h}$. The nuclear proteins (A) and cytoplasmic proteins (B) were extracted as described in Materials and Methods. The levels of Foxo3 were determined by western blotting analysis. $\beta$-tubulin was used as cytoplasmic marker and lamin B1 was used as nuclear marker in RC-4B/C cells The gray values of Foxo3a was shown in histogram in different concentrations of matrine-treated pituitary cancer cells. ${ }^{* *} \mathrm{P}<0.01$, compared with RC-4B/C cells without matrine.

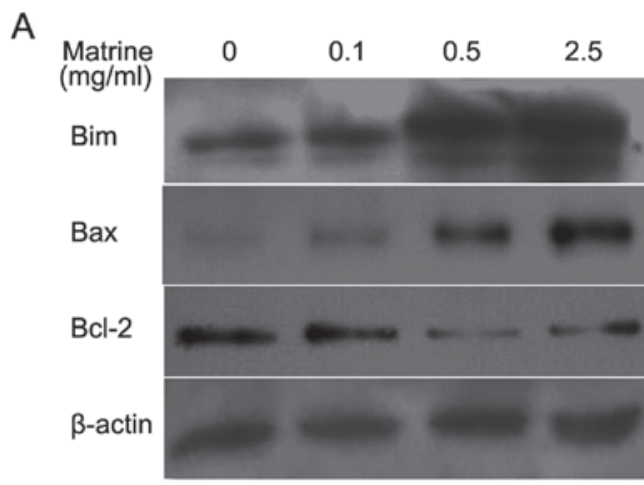

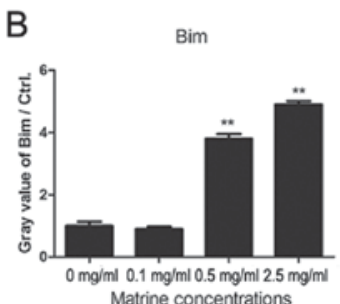
Matrine concentrations

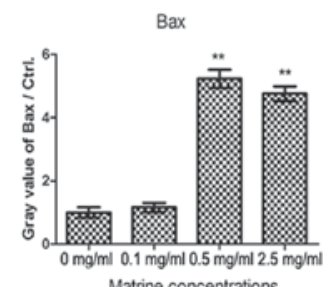

Matrine concentrations

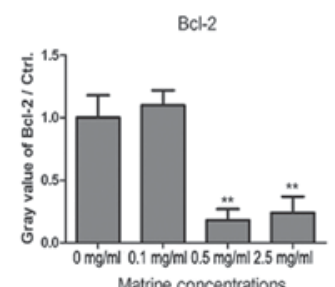

Matrine concentrations

Figure 4. Matrine increases the expression of pro-apoptotic proteins in RC-4B/C cells. (A) RC-4B/C cells were treated with $0.1,0.5 \mathrm{and} 2.5 \mathrm{mg} / \mathrm{ml}$ of matrine for $24 \mathrm{~h}$ and the expression of Bim, Bax and Bcl-2 was determined by western blotting analysis. RC-4B/C cells treated with $0.1 \%$ DMSO were used as negative controls. $\beta$-actin was used as internal reference gene in pituitary cancer cells. (B) The gray values of Bim, Bax and Bcl-2 were shown in histogram of matrine-treated $\mathrm{RC}-4 \mathrm{~B} / \mathrm{C}$ cells. ${ }^{* *} \mathrm{P}<0.01$, compared with $\mathrm{RC}-4 \mathrm{~B} / \mathrm{C}$ cells without matrine.

normal and neoplastic pituitary cells show a differential sensitivity to matrine. But, we had tested the role of Matrine in 'normal' liver cell line-L02 cells. The results demonstrated that Matrine had showed a dual effect on the growth of 
normal cells L02. At a lower concentration $(0.1 \sim 0.4 \mathrm{mg} / \mathrm{ml})$ of Matrine, the proliferation of L02 cells were significantly increased, especially being treated for $72 \mathrm{~h}$. However, higher concentration of Matrine (more than $0.8 \mathrm{mg} / \mathrm{ml}$ ) showed an increasing dose-dependent cytotoxicity in L02 cells (the data was unpublished). All the data demonstrated that lower concentration of Matrine might promote the cell proliferation of normal cells and higher concentration of Matrine could kill normal cells as well. Thus, it is an interesting question that how to increase the sensitivity of tumor cells to matrine but decrease the cytotoxic effects on normal cells, that would be a perfect anti-tumor reagent in pituitary tumor therapy. Intracranial pituitary tumors are generally small in size, deep in position, and not easy to observe and measure; moreover, the variability of tumor growth and the large tumor often oppress the lower part of the hypothalamus, which usually causes sudden death of the animal. Therefore, next, we would construct animal model of human transplantable pituitary tumor in nude mice by subcutaneous transplantation and treat them with different doses of Matrine to observe the dynamic change of tumor volume, and take the tumor tissue for molecular In-depth study of mechanism. This would help to further prove the anti-tumor effects of matrine. In conclusion, matrine induced cell death of pituitary cancer cells and the molecular mechanism results showed that the inhibition of cell proliferation might be involved in Akt/Foxo3a signaling pathway. Matrine could be used as a novel agent in clinical therapy of pituitary cancer patients.

\section{Acknowledgements}

Not applicable.

\section{Funding}

No funding was received.

\section{Availability of data and materials}

All data generated or analyzed during the present study are included in this published article.

\section{Authors' contributions}

LL and PG performed the literature analysis and designed the experiment. LL and SC performed the experimental studies. YS and LK performed data analysis. GL and LK were involved in the acquisition and interpretation of data.

\section{Ethics approval and consent to participate}

Not applicable.

\section{Patient consent for publication}

Not applicable.

\section{Competing interests}

The authors declare that they have no competing interests.

\section{References}

1. Zhou HJ, Zhan RY, Ma YH, Cao F and Zheng XJ: Primary sellar melanocytic tumor mimicking hemorrhagic pituitary macroadenoma: Case report and literature review. Br J Neurosurg 29: 298-302, 2015.

2. Loyo-Varela M, Herrada-Pineda T, Revilla-Pacheco F and Manrique-Guzman S: Pituitary tumor surgery: Review of 3004 cases. World Neurosurg 79: 331-336, 2013.

3. Nasi D, Perano D, Ghadirpour R, Iaccarino C, Servadei F and Romano A: Primary pituitary neuroendocrine tumor: Case report and literature review. Surg Neurol Int 8: 101, 2017.

4. Wang W, Fu L, Li S, Xu Z and Li X: Histone deacetylase 11 suppresses p53 expression in pituitary tumor cells. Cell Biol Int 41: 1290-1295, 2017.

5. Zhao ZR, Li M, Shi P and Zhang P: MiRNA153 induces pituitary tumor MMQ cell line apoptosis through down-regulating Skp protein expression. Eur Rev Med Pharmacol Sci 21: 1270-1275, 2017.

6. Liu B, Pang B, Wang Q, Yang S, Gao T, Ding Q, Liu H, Yang Y, Fan $\mathrm{H}$, Zhang R, et al: EZH2 upregulation correlates with tumor invasiveness, proliferation, and angiogenesis in human pituitary adenomas. Hum Pathol 66: 101-107, 2017.

7. Chang C, Liu SP, Fang CH, He RS, Wang Z, Zhu YQ and Jiang SW: Effects of matrine on the proliferation of HT29 human colon cancer cells and its antitumor mechanism. Oncol Lett 6: 699-704, 2013.

8. Li H, Li X, Bai M, Suo Y, Zhang G and Cao X: Matrine inhibited proliferation and increased apoptosis in human breast cancer MCF-7 cells via upregulation of Bax and downregulation of Bcl-2. Int J Clin Exp Pathol 8: 14793-14799, 2015.

9. Yu P, Liu Q, Liu K, Yagasaki K, Wu E and Zhang G: Matrine suppresses breast cancer cell proliferation and invasion via VEGF-Akt-NF-kappaB signaling. Cytotechnology 59: 219-229, 2009.

10. Luo C, Zhong HJ, Zhu LM, Wu XG, Ying JE, Wang XH, Lü WX, $\mathrm{Xu}$ Q, Zhu YL and Huang J: Inhibition of matrine against gastric cancer cell line MNK45 growth and its anti-tumor mechanism. Mol Biol Rep 39: 5459-5464, 2012.

11. Luo C, Zhu Y, Jiang T, Lu X, Zhang W, Jing Q, Li J, Pang L, Chen K, Qiu F, et al: Matrine induced gastric cancer MKN45 cells apoptosis via increasing pro-apoptotic molecules of Bcl-2 family. Toxicology 229: 245-252, 2007.

12. Zhang J, Li Y, Chen X, Liu T, Chen Y, He W, Zhang Q and Liu S: Autophagy is involved in anticancer effects of matrine on SGC-7901 human gastric cancer cells. Oncol Rep 26: 115-124, 2011.

13. Li H, Xie S, Liu X, Wu H, Lin X, Gu J, Wang H and Duan Y: Matrine alters microRNA expression profiles in SGC-7901 human gastric cancer cells. Oncol Rep 32: 2118-2126, 2014.

14. An Q, Han C, Zhou Y, Li F, Li D, Zhang X, Yu Z, Duan Z and Kan Q: Matrine induces cell cycle arrest and apoptosis with recovery of the expression of miR-126 in the A 549 non-small cell lung cancer cell line. Mol Med Rep 14: 4042-4048, 2016.

15. Egawa H, Jingushi K, Hirono T, Ueda Y, Kitae K, Nakata W, Fujita K, Uemura M, Nonomura N and Tsujikawa K: The miR-130 family promotes cell migration and invasion in bladder cancer through FAK and Akt phosphorylation by regulating PTEN. Sci Rep 6: 20574, 2016.

16. Lin CH, Chang CY, Lee KR, Lin HJ, Chen TH and Wan L: Flavones inhibit breast cancer proliferation through the Akt/FOXO3a signaling pathway. BMC Cancer 15: 958, 2015.

17. Gao H, Guo Y, Deng N, Fei P, Qiu X, Zheng P, Feng J and Dai G: Suppressive effect of matrine on tumor invasion in N-Butyl-N-(4-Hydroxybutyl)Nitrosamine-induced urinary bladder carcinogenesis. Chemotherapy 60: 119-128, 2014.

18. Bai S, Chen T, Yu X, Luo M, Chen X, Lin C, Lai Y and Huang H: The specific killing effect of matrine on castration-resistant prostate cancer cells by targeting the Akt/FoxO3a signaling pathway. Oncol Rep 37: 2819-2828, 2017.

19. Li LQ, Li XL, Wang L, Du WJ, Guo R, Liang HH, Liu X, Liang DS, Lu YJ, Shan HL and Jiang HC: Matrine inhibits breast cancer growth via miR-21/PTEN/Akt pathway in MCF-7 cells. Cell Physiol Biochem 30: 631-641, 2012.

20. Tang M, Zhao Y, Liu N, Chen E, Quan Z, Wu X and Luo C: Overexpression of HepaCAM inhibits bladder cancer cell proliferation and viability through the AKT/FoxO pathway. J Cancer Res Clin Oncol 143: 793-805, 2017.

21. Mu DW, Guo HQ, Zhou GB, Li JY and Su B: Oleanolic acid suppresses the proliferation of human bladder cancer by $\mathrm{Akt} / \mathrm{mTOR} / \mathrm{S} 6 \mathrm{~K}$ and ERK1/2 signaling. Int J Clin Exp Pathol 8: 13864-13870, 2015. 